\section{Ventilación mecánica no invasiva como estrategia adyuvante en el manejo del fallo respiratorio agudo secundario a edema pulmonar periparto por preeclampsia severa}

\section{Non-invasive mechanical ventilation as adjuvant strategy in the management of acute respiratory failure secondary to peripartum pulmonary edema in severe preeclampsia}

\section{Sr. Director:}

El edema agudo de pulmón (EAP) es una enfermedad muy rara durante el embarazo, con una frecuencia de un $0,08 \%$ de los casos, entre las principales causas relacionadas se encuentran el tratamiento con tocolíticos, enfermedades cardíacas preexistentes, terapia de reposición de líquidos y preeclampsia ${ }^{1}$. En el contexto de la preeclampsia se puede presentar hasta en un $2,9 \%$ de los casos, con una mayor incidencia en multigrávidas y mujeres de mayor edad ${ }^{2}$.

Dentro de las alteraciones hemodinámicas que intervienen en el desarrollo del edema pulmonar en las pacientes preeclámpticas tenemos: elevación de la presión hidrostática vascular, incremento de la permeabilidad capilar y disminución de la presión oncótica ${ }^{3}$. En la preeclampsia, estas alteraciones no sólo son más pronunciadas, sino que se asocian a un aumento de la poscarga, un funcionamiento cardíaco hiperdinámico y la presencia de disfunción renal que, en conjunto, predisponen al desarrollo de EAP en este tipo de pacientes ${ }^{4}$, situación potencialmente mortal para la madre y el feto ${ }^{2}$.

La ventilación mecánica no invasiva (VMNI) es una estrategia alternativa para proporcionar apoyo ventilatorio a pacientes con insuficiencia respiratoria aguda, lo cual resulta en una reducción de la necesidad de intubación orotraqueal (IOT) y mortalidad hospitalaria ${ }^{5}$. Un reciente metaanálisis mostró que la aplicación de la VMNI en el contexto de EAP cardiogénico reduce la necesidad de IOT y la mortalidad de manera significativa ${ }^{6}$. Sin embargo, su uso en el embarazo es poco común, la mayoría de la información se limita a reporte y series de casos. No existe en nuestro conocimiento reportes previos de esta estrategia ventilatoria en pacientes con falla respiratoria inducida por preeclampsia, por lo que el objetivo del presente reporte es presentar la experiencia de este modo ventilatorio como una potencial indicación para el manejo de esta enfermedad.

Se presenta el caso de una mujer de 25 años de edad, primigesta, admitida en la Clínica Maternidad Rafael Calvo de Cartagena, Colombia, por cuadro de preeclampsia severa, con presiones arteriales > 160/110 $\mathrm{mmHg}$, manejada con sulfato de magnesio y labetalol intravenoso sin obtener adecuada respuesta, por lo que se realiza cesárea, que culmina con recién nacido sano, se traslada posteriormente a unidad de cuidados intensivos ( $\mathrm{UCl}$ ) para manejo con infusión de vasodilatadores y vigilancia neurológica por riesgo elevado de eclampsia.

Al ingreso en $\mathrm{UCl}$ presentó presión arterial de 161/ $120 \mathrm{mmHg}$, taquicardia, inadecuado patrón respiratorio, saturación de oxígeno arterial $\left(\mathrm{SaO}_{2}\right)$ del $89 \%$, crepitantes bibasales y presión venosa central (PVC) de $16 \mathrm{mmHg}$. Se realiza radiografía de tórax que muestra cefalización del flujo, con hilios pulmonares congestivos; se considera entonces el diagnóstico de EAP. Se inicia manejo con oxígeno por sistema Ventury al $50 \%$, cabecera a $45^{\circ}$, furosemida y nitroglicerina intravenosa.

A pesar del manejo con vasodilatadores, persiste hipertensa, motivo por el cual se adiciona al manejo medicamentos antihipertensivos orales. En el contexto de una paciente con EAP refractario a tratamiento, se decide iniciar VMNI en modo presión positiva continua en vía aérea (CPAP), para mejorar el trabajo respiratorio, revertir la hipoxemia y evitar la IOT. Se utilizó mascarilla oronasal para reducir las fugas y favorecer la adaptación de la paciente.

La paciente estuvo en VMNI durante 3 días, con mejora progresiva de los parámetros ventilatorios y gasométricos, llegando a la estabilidad hemodinámica sin necesidad de soporte ventilatorio invasivo, suspensión de vasodilatadores intravenosos, con lo que se logró dar de alta de cuidados intensivos con antihipertensivos orales y posterior alta hospitalaria al séptimo día de su ingreso.

Aunque la mayor preocupación de la VMNI en el embarazo es el riesgo de broncoaspiración debido al aumento en la presión abdominal, no existe una recomendación basada en la evidencia que contraindique el uso de la VMNI en obstetricia. Nuestra paciente no presentó ninguna alteración asociada al uso de esta terapia, con un resultado similar al encontrado en algunos reportes de condiciones patológicas similares en pacientes obstétricas, por ejemplo, Terajima et al utilizaron la VMNI en combinación con anestesia 
epidural y espinal durante la cesárea de una parturienta a las 23 semanas de gestación que presentó edema pulmonar e hipoxia, obteniendo buenos resultados maternofetales ${ }^{7}$. Fuentes et al comunicaron un caso de una paciente con hipertensión gestacional y obesidad mórbida que a las 24 horas del posparto desarrolló EAP con deterioro de la función respiratoria, iniciándose VMNI con mejoría clínica ${ }^{8}$. Igual resultado se obtuvo mediante la aplicación de VMNI en el manejo del EAP secundario a tocolíticos durante el parto de un embarazo gemelar ${ }^{9}$.

Aunque en este reporte, esta terapia fue aplicada en el posparto, los resultados maternos pueden ser comparables entre mujeres en embarazo y en posparto inmediato, puesto que la fisiología pulmonar materna permanece con un comportamiento similar incluso hasta semanas posparto ${ }^{10}$.

La VMNI con presión positiva puede ser útil en el tratamiento del EAP refractario por preeclampsia severa. En nuestra opinión, constituye una opción potencial y segura que tener en cuenta en pacientes seleccionadas y bajo una monitorización adecuada. En el caso presentado, el tratamiento adyuvante con VMNI contribuyó a evitar la IOT de la paciente y mejoró sustancialmente la evolución en pocos días. Esta terapia debe someterse a la investigación clínica para determinar su utilidad e inferencias en la morbimortalidad de estas pacientes.

\section{Agradecimientos}

A la paciente, por su permiso para la publicación del caso, y al equipo de trabajo de la unidad de cuidados intensivos Gestión Salud IPS, por su colaboración en el manejo de esta y otras pacientes obstétricas críticamente enfermas.

\section{Bibliografía}

1. Sciscione AC, Ivester T, Largoza M, Manley J, Shlossman $P$, Colmorgen $\mathrm{GH}$. Acute pulmonary edema in pregnancy. Obstet Gynecol. 2003;101:511-5.

2. Sibai BM, Mabie WC, Harvey CJ, Gonzalez AR. Pulmonary edema in severe preeclampsia-eclampsia: Analysis of 37 consecutive cases. Am J Obstet Gynecol. 1987;156:1174-9.
3. Benedetti TJ, Kates R, Williams V. Hemodynamic observations in severe preeclampsia complicated by pulmonary edema. Am J Obstet Gynecol. 1985;152:330-4.

4. Miranda JL, Martí-Viaño. Cuidados intensivos de las complicaciones obstétricas y del embarazo. En: Torres, LM, editor. Tratado de Cuidados Críticos y Emergencias. Editorial Aran Ediciones, S.A. 2002. p. 1419-1446.

5. Ho RP, Boyle M. Non-invasive positive pressure ventilation in acute respiratory failure: providing competent care. Aust Crit Care. 2000;13:135-7.

6. Weng CL, Zhao YT, Liu QH, Fu CJ, Sun F, Ma YL, et al. Metaanalysis: Noninvasive ventilation in acute cardiogenic pulmonary edema. Ann Intern Med. 2010;152:590-600.

7. Terajima K, Suzuki R, Suganuma R, Sakamoto A. Non-invasive positive pressure ventilation and subarachnoidal blockade for Caesarean section in a parturient with pulmonary edema. Acta Anaesthesiol Scand. 2006;50:1307-8.

8. Fuentes Pradera MA, Suárez Delgado JM, Suan Rodríguez C, Yanes Vidal G, Pajuelo Gallego A. Papel de la ventilación mecánica no invasiva en un caso de edema agudo pulmonar de una paciente puérpera obesa mórbida. Rev Esp Anestesiol Reanim. 2005;52:122-3.

9. Perbet S, Constantin JM, Bolandard F, Vignaud M, Gallot D, Chanséaume $S$, et al. Non-invasive ventilation for pulmonary edema associated with tocolytic agents during labour for a twin pregnancy. Can J Anaesth. 2008;55:769-73.

10. Bobrowski RA. Pulmonary physiology in pregnancy. Clin Obstet Gynecol. 2010;53:285-300.

J. Rojas-Suarez ${ }^{\mathrm{a}, \mathrm{b}, *}$, M. Cogollo-González ${ }^{\mathrm{b}}$, M.C. García-Rodríguez ${ }^{\mathrm{b}}$, A. Paternina-Caicedo ${ }^{\mathrm{a}}$

y J. Miranda-Quintero ${ }^{a}$

a Universidad de Cartagena, Facultad de Medicina, Cartagena, Colombia

b Unidad de Cuidados Intensivos, Gestión Salud S.A., Clínica Maternidad Rafael Calvo, Cartagena, Colombia

* Autor para correspondencia.

Correo electrónico: jocherojas2005@hotmail.com (J. Rojas-Suarez).

doi:10.1016/j.medin.2011.01.004

\section{Tratamiento del síndrome de Kounis}

\section{Treatment of Kounis syndrome}

Varón de 54 años, sin historia de alergias. Presenta como antecedentes hipertensión arterial (HTA), obesidad y tabaquismo. Acude a urgencias por dolor abdominal en fosa ilíaca derecha y fiebre; Blumberg positivo. Se administró analgesia y amoxicilina-clavulánico intravenoso (i.v.). A los 10 min de inicio del antibiótico se observa rash generalizado, shock y dolor retroesternal opresivo. El electrocardiograma (ECG) muestra elevación del segmento ST mayor de $3 \mathrm{~mm}$ en derivaciones inferiores. Se suspende el antibiótico, y se administran corticoides, antihistamínicos, $\mathrm{O}_{2}$ al $50 \%$, morfina y antiagregantes. Precisó apoyo con volumen y dopamina, junto con antagonistas $\mathrm{H}_{1}\left(\right.$ anti- $\left.\mathrm{H}_{1}\right)$ y anti- $\mathrm{H}_{2}$, continuándose con pauta de hidrocortisona y antibioterapia empírica con ciprofloxacino y metronizadol. Aproximadamente a los 15 min de instaurado el tratamiento, cede el dolor torácico y el segmento ST desciende más del $50 \%$, corrigiéndose a las $3 \mathrm{~h}$. Se retiró el apoyo simpaticomimético precozmente, presentando HTA, iniciándose nitritos i.v. e IECA. Se pospuso la cirugía abdominal a las $41 \mathrm{~h}$ de evolución por manifestaciones hemorrágicas menores. Se añadieron posteriormente antagonista del calcio y bloqueadores beta (BB). La CK-MB masa máxima fue 35,3; troponina T máxima, $0,63 \mathrm{ng} / \mathrm{ml}$. La coronariografía mostró enfermedad de un vaso: estenosis del $90 \%$ de primera diagonal con mal lecho y dominancia derecha. El diagnóstico es un síndrome de Kounis tipo II, con la particularidad de que la coronariografía mostró una lesión en una arteria distinta de donde se produjo el evento isquémico. 